\title{
DOCEAMUS
}

\author{
doceamus ... let us teach
}

\section{Students' Combinatorial \\ Reasoning: Counting Processes and Sets of Outcomes}

\section{Elise Lockwood}

\author{
Communicated by Benjamin Braun
}

My research focuses on undergraduate students' reasoning about combinatorics, and this note specifically concerns "counting problems" - determining the cardinalities of sets that satisfy certain conditions. These problems involve a wide variety of reasoning skills, but my major findings all elaborate a central theme:

Students should focus on the sets of outcomes they are trying to count, not just the counting process.

Although it might be clear to mathematicians that counting is fundamentally focused on sets of outcomes, this is not always how students view counting; nor is it always how students are taught to count in university courses. My goal here is to share highlights from my research on teaching discrete mathematics, combinatorics, and probability.

In 2013 I [1] introduced a model of students' combinatorial reasoning with three interconnected components: formulas/expressions, counting processes, and sets of outcomes (Figure 1). Formulas/expressions are mathematical expressions that yield numerical values that are often considered the "answer" to a counting problem, for example $\left(\begin{array}{l}8 \\ 5\end{array}\right) \cdot\left(\begin{array}{l}4 \\ 3\end{array}\right)$ or $\frac{9 !}{2 !}-8$. Counting processes are the enumeration processes in which a counter engages as they solve a counting problem. For example, a counting process to enumerate all arrangements of the letters A, B, C, and

Elise Lockwood is assistant professor of mathematics at Oregon State University. Her email address is e1ise.1ockwood@ oregonstate. edu.

For permission to reprint this article, please contact: reprint-permission@ams .org.

DOI: http://dx.doi.org/10.1090/noti1705
$\mathrm{D}$ might involve using the multiplication principle in a four-stage process of first considering which letter can be in the first position, then considering which letter can be second, then third, and then fourth. Sets of outcomes are the collections of objects being counted, and these can often be organized in a way that reflects the counting process used.

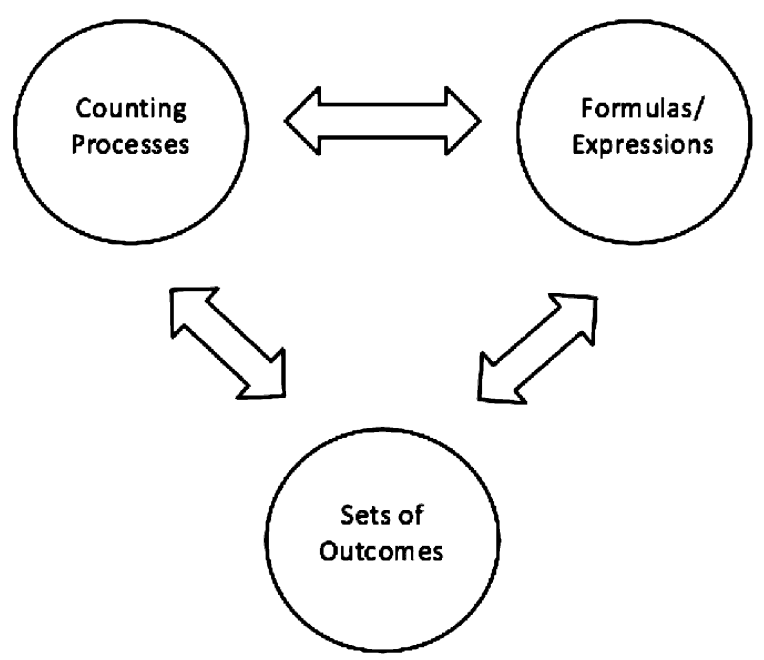

Figure 1: Lockwood's model of students' combinatorial thinking has three interconnected components $[1,5]$. 
In my own experience, it has been surprising to see how frequently students attempt to solve counting problems without giving explicit attention to what they are trying to count. I use the phrase set-oriented perspective toward counting to refer to the "way of thinking about counting that involves attending to sets of outcomes as an intrinsic component of solving counting problems" [2, p. 31]. My research suggests that by explicitly teaching students to develop a set-oriented perspective toward counting, instructors can achieve the following three goals.

1. Help students reason about common problematic issues that arise in counting, particularly determining whether or not order matters and identifying/correcting overcounting. Sets of outcomes can help to illuminate for students common errors in solely process-based reasoning. For example, the phrase "order matters" (which is not always well understood by students) means that one needs to consider the nature of the outcomes, whether the problem is counting sets or sequences, for example. For problems whose outcomes are sets, order "does not matter" because different arrangements of elements within the sets do not create distinct outcomes. Students who focus on the nature of what is being counted to determine whether order matters may gain a better understanding of their solution methods than those who merely attempt to fit given formulas to certain kinds of problems.

Students can also use the relationship between counting processes and sets of outcomes to reason about overcounting. To help students see this, instructors can give problems in which overcounting naturally occurs and have students reflect on how counting processes generate too many outcomes. The following problem (adapted from Tucker [4]) is a favorite of mine to introduce how a counting process can overcount a set of outcomes.

How many ways are there to form a three-letter sequence using the letters $a, b, c, d, e, f$ :

(a) without repetition and containing the letter $e$ ?

(b) with repetition and containing at least one $e$ ?

Part (b) has a common incorrect answer of $3 \cdot 6 \cdot 6$. In order to explain why this answer does not work, students need to very clearly understand how the counting process reflected by $3 \cdot 6 \cdot 6$ generates too many outcomes. Focusing on the relationship between counting processes and sets of outcomes can help to illuminate the phenomenon of overcounting.

2. Help students understand the relationship between counting processes and sets of outcomes through the use of explicit listing. We want students to be able to explicitly generate and structure sets of outcomes to explain their reasoning. B. Gibson and I [3] found a positive correlation between listing outcomes and solving problems correctly among novice undergraduates. Our findings suggest that creating explicit lists is a worthwhile activity that grounds students' thinking and activity in sets of outcomes, even though students might find it mundane. Students will necessarily need to move on to more efficient methods and formulas, but initial listing activity can help them orient themselves to what they are trying to count. Furthermore, by understanding the relationship between counting processes and sets of outcomes, students can become more flexible counters, who are willing to consider alternate processes and problem-solving approaches.

3. Help students justify key formulas. Too often we present students with formulas for basic combinatorial operations, and they struggle to make sense of them even when a combinatorial explanation has been provided. This is indicative of a broader phenomenon in mathematics in which students may not understand formulas, but it feels especially relevant in combinatorics, where a few key formulas form the basis for much of counting. My research has found that students can make sense of, justify, and even develop formulas if they are given opportunities to reason about counting processes and sets of outcomes. Specifically, one idea is for instructors to give students a number of problems that involve different simple combinatorial operations (such as some permutation problems and certain combination problems), without first having presented formulas. Then, have students solve then categorize those problems and articulate what each kind of problem counts. Through this activity, students can engage in problem solving, reflect on their processes, and consider the nature of what they are trying to count. Students can either then generalize a formula themselves, or, if they are then presented with a formula, they can have a better understanding of why a formula makes sense, why an identity holds, or why a solution is correct. This development of student knowledge based on problem-solving stands in stark contrast to a scenario in which students are provided with a formula and then asked to apply that formula repeatedly.

\section{References}

[1] Lockwood, E. (2013). A model of students' combinatorial thinking. J. Math. Behavior, 32, 251-265. DOI: 10.1016/j. jmathb.2013.02.008.

[2] LOCKWOOD, E. (2014). A set-oriented perspective on solving counting problems. For the Learning of Mathematics, 34(2), 31-37.

[3] Lockwood, E., AND GiBson, B. (2016). Combinatorial tasks and outcome listing: Examining productive listing among undergraduate students. Ed. Stud. in Math., 91(2), 247-270. DOI: 10.1007/s10649-015-9664-5.

[4] Tucker, A. (2002). Applied Combinatorics (4th ed.). New York: John Wiley \& Sons.

[5] Lockwood, E., Reed, Z., And Caughman, J. S. (2017). An analysis of statements of the multiplication principle in combinatorics, discrete, and finite mathematics textbooks. Int. J. of Res. in Und. Math. Ed., 3(3), 381-416. DOI: 10.1007/ s40753-016-0045-y.

\section{Image Credits}

Figure 1 Reprinted by permission from Springer: Springer Nature, International Journal for Research in Undergraduate Mathematics Education, "An Analysis of Statements of the Multiplication Principle in Combinatorics, Discrete, and Finite Mathematics Textbooks," Elise Lockwood, Zackery Reed, John S. Caughman, (c) 2016.

Author photo courtesy of the College of Science at Oregon State University. 


\section{ABOUT THE AUTHOR}

Elise Lockwood loves research and feels fortunate to be able to teach students how to count. She recently received an NSF Early Career award to study students' combinatorial reasoning in computational settings. She enjoys running, cooking, traveling, collecting Rocket Raccoon comics, and playing with her Ragdoll

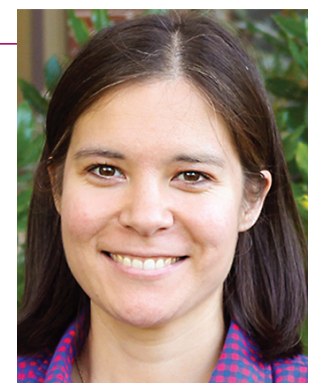

Elise Lockwood cats, Nicodemus and Sebastian.

\section{Recent volumes from MSJ}

Advanced Studies in Pure Mathematics http://mathsoc.jp/publication/ASPM/

Volume 75

\section{Algebraic Varieties and Automorphism Groups}

Edited by K. Masuda (Kwansei Gakuin),

T. Kishimoto (Saitama),

H. Kojima (Niigata),

M. Miyanishi (Kwansei Gakuin),

M. Zaidenberg (Grenoble Alpes)

ISBN 978-4-86497-048-8

Volume 74

Higher Dimensional

Algebraic Geometry in honour of Professor Yujiro Kawamata's sixtieth birthday

Edited by K. Oguiso (Tokyo), C. Birkar (Cambridge), S. Ishii (Tokyo Woman's Christian), S. Takayama (Tokyo) ISBN 978-4-86497-046-4

Volume 73

Hyperbolic Geometry and

Geometric Group Theory

Edited by K. Fujiwara (Kyoto), S. Kojima (Tokyo Tech),

K. Ohshika (Osaka)

ISBN 978-4-86497-042-6

MSJ Memoirs

http://mathsoc.jp/publication/memoir/memoirs-e.html

Volume 36

Global solutions and

the asymptotic

behavior for nonlinear wave equations with

small initial data

Soichiro Katayama

ISBN 978-4-86497-054-9

Volume 35

Foundations of

the minimal model program

Osamu Fujino

ISBN 978-4-86497-045-7

$\nabla \nabla \nabla$ For purchase, visit $\nabla \nabla \nabla$

http://www.ams.org/bookstore/aspmseries

http://www.worldscientific.com/series/aspm

http://www.worldscibooks.com/series/msjm

The Mathematical Society of Japan 34-8, Taito 1-chome, Taito-ku

Tokyo, JAPAN

http://mathsoc.jp/en/ 